\title{
Defective resin composite - repair or replace?
}

\author{
Abstracted from \\ Sharif MO, Catleugh M, Merry A, Tickle M, Dunne SM, Brunton P, Aggarwal VR. \\ Replacement versus repair of defective restorations in adults: resin composite. \\ Cochrane Database Syst Rev. 2010 issue 2. \\ Address for correspondence: Luisa Fernandez Mauleffinch, Review Group Co-ordinator, \\ Cochrane Oral Health Group, MANDEC, School of Dentistry, University of Manchester, \\ Higher Cambridge Street, Manchester M15 6FH, UK. E-mail: luisa.fernandez@manchester.ac.uk
}

\section{Question: In adult patients with defective resin composite restorations is repair with resin composite more effective than replacement?}

Data sources Cochrane Oral Health Group Trials Register, the Cochrane Central Register of Controlled Trials (CENTRAL), Medline, Embase, ISI Web of Science, ISI Web of Science Conference Proceedings, BIOSIS, OpenSIGLE. Reference lists of all eligible trials and review articles and their reference lists were searched.

Study selection Trials were selected if they met the following criteria: randomised or quasi-randomised controlled trial, involving replacement and repair of resin composite restorations.

Data extraction and synthesis Titles and abstracts were assessed independently by two review authors for relevance. Full papers were obtained for relevant articles and both review authors studied these. Data synthesis was to follow Cochrane Collaboration statistical guidelines.

Results 279 potentially eligible studies were identified. Only four studies were analysed further but none of these met the inclusion criteria and all were excluded from this review.

Conclusions There are no published randomised controlled clinical trials relevant to this review question. There is therefore a need for methodologically sound randomised controlled clinical trials that are reported according to the Consolidated Standards of Reporting Trials (CONSORT) statement (www.consort-statement.org/). Further research also needs to explore qualitatively the views of patients on repairing versus replacement and investigate themes around pain, distress and anxiety, time and costs.
This paper is based on a Cochrane Review published in the Cochrane Library 2010, issue 2 (see www.thecochranelibrary.com for information). Cochrane Reviews are regularly updated as new evidence emerges and in response to feedback, and the Cochrane Library should be consulted for the most recent version of the review.

\section{Commentary}

This well thought out, focused and organised Cochrane systematic review found no high level quality evidence to match their very narrow inclusion criteria with regard to the successful (effective) repair of defective composite restorations with composite, as compared to complete replacement with composite in permanent molar and premolar teeth.

A broad electronic database search was performed and efforts made to find relevant unpublished articles. The articles selected were assessed by more than one reviewer. None of the 279 potentially eligible articles found met the authors' very narrow inclusion criteria (randomised clinical trials, quasi-randomised clinical trials, including split mouth studies), and therefore no articles were finally selected for the review. The remainder of the review commented on potential research pathways that could lead to successful high level quality results and their subsequent interpretation strategy.

As the authors appropriately suggested, 'methodologically sound randomised clinical trials' with CONSORT style reporting would necessary in order to meaningfully test the authors' null hypothesis on this subject utilising their inclusion criteria. However, such long term clinical trials with statistically and clinically meaningful results may be difficult and costly to accomplish.

The authors do suggest, based on the articles retrieved, 'that repair of restorations could be effective and the survival rate at two-year follow-up is good'. Based on the limitations brought out by this review, utility in practice decisions must be used with caution in conjunction with clinical experience, professional judgment, and patient informed consent. Even in the hands the most skillful practitioner, there is no doubt that the entire replacement of a restoration requires the removal of more tooth structure and therefore, the possibility exists of having post-operative sensitivity as an unwanted side effect of this 'surgical intervention'. Repair or refurbishment may be an alternative when the restoration is assessed and diagnosed appropriately and that the repair will not affect the future wellbeing of the tooth and the damaged restoration.

Globally, dental practitioners are dealing with the situations outlined in the review on a routine basis. Variations in clinical practice are very common. A study conducted by one of the Practice Based Research Networks (DPBRN) ${ }^{1}$ in the USA, evaluating the practitioner's attitudes towards the diagnosis, repair, replacement, or refurbishment of restorations, had very interesting findings. For the same clinical scenarios presented to the participants in a survey, from the 512 participants the authors found that:

- Younger practitioners seem to be more conservative than older practitioners; possibly as a result of greater exposure to different 


\section{RESTORATIVE DENTISTRY}

diagnostic tools and greater emphasis on risk assessment and prevention than previous generations.

- European practitioners in the study seem to be more conservative than their counterpart Americans.

- Practices had their revenues and costs linked to procedures being done which were more reflective of the complete removal and replacement of the broken restoration.

In conclusion, the findings from this systematic review are extremely limited. From the authors' discussion of weak evidence obtained from four clinical trials that did not meet their very narrow inclusion criteria, a more conservative approach to total replacement may be a consideration. This would also seem to be in line with the apparent emerging global consensus of conservative invasive treatment in lieu of 'extension for prevention'. More high level evidence supporting studies are needed.

\section{Practice points}

- Defective composite restorations may be repaired with composite materials instead of a complete removal of the restoration the decision needs to be made after a thorough evaluation of presenting circumstances.

Peter Mychajiliw and Analia V Keenan NYU College of Dentistry, New York, USA.

1. Gordan W, Garvan CW, Richman JS, et al., DPBRN Collaborative Group. How dentists diagnose and treat defective restorations: evidence from the dental practice-based research network. Oper Dent 2009; 34: 664-673.

Evidence-Based Dentistry (2010) 11, 112-113. doi:10.1038/sj.ebd.6400757 